\title{
Transformation of Holes Emission Paths under Negative Bias Temperature Stress in Deeply Scaled pMOSFETs
}

\author{
Yiming Liao, ${ }^{1}$ Xiaoli Ji, ${ }^{1}$ Qiang Guo, ${ }^{2}$ and Feng Yan ${ }^{1}$ \\ ${ }^{1}$ School of Electronics Science and Engineering, Nanjing University, No. 163 Xianlin Road, Nanjing, Jiangsu 210046, China \\ ${ }^{2}$ Quality and Reliability Engineering, Wuhan Xinxin Semiconductor Manufacturing Company, No. 18 Gaoxinsi Road, \\ Wuhan, Hubei 430205, China \\ Correspondence should be addressed to Feng Yan; fyan@nju.edu.cn
}

Received 29 October 2014; Revised 6 November 2014; Accepted 7 November 2014

Academic Editor: Jiwu Lu

Copyright (C) 2015 Yiming Liao et al. This is an open access article distributed under the Creative Commons Attribution License, which permits unrestricted use, distribution, and reproduction in any medium, provided the original work is properly cited.

\begin{abstract}
We examine the impact of negative bias temperature (NBT) stress on the fluctuations in $I_{D}$ and $I_{G}$ for deeply scaled pMOSFETs and find that the relative high NBT stress triggers $I_{G}$-RTN and $I_{D}$-step. Through the analysis of the field dependence of emission constant and the carrier separation measurement, it is found that under the relative high NBT stress some traps keep charged state for very long time, as observing step-like behaviors in $I_{D}$, while other traps emit charged holes to the gate side through TAT process, which originate both $I_{D}$-step and ID-RTN.
\end{abstract}

\section{Introduction}

Negative bias temperature instability (NBTI) degradation is one of the most important reliability issues in modern complementary metal-oxide-semiconductor (CMOS) technologies [1-7]. Recently, much attention has been paid to NBTS generated oxide traps, which could significantly increase the failure probability in deeply scaled pMOSFETs. Furthermore, these traps are currently considered to not only cause the $V_{\text {th }}$ degradation but also increase the gate leakage current $\left(I_{G}\right)$ [8-10]. However, someone considers the $I_{G}$ fluctuations are probably not to be explained as the results of NBT induced switching traps [11-13]. Tsujikawa reported that the observed transient $I_{G}$ and $I_{D}$ signal simultaneously in small size pMOS devices are not linked directly with each other [11]. Wagner et al. [12] pointed out that the increases of $I_{G}$ fluctuation are probably caused by the beginning of oxide breakdown which should not be explained as the results of NBT induced switching trap while Gao et al. [14] have reported that $I_{G}$ increases only when part of the switching hole traps transform to the permanent bulk traps with increasing NBT stress or stressing time. To date, the impact of negative bias temperature stress condition on $I_{D}$ and $I_{G}$ fluctuation has not been systematically evaluated yet.

In this paper, we examine the impact of stress on the $I_{G^{-}}$ RTN and $I_{D}$-RTN for deeply scaled pMOSFETs, using carrier separation measurement to identify the type of $I_{G}$-RTN. The characteristic of time constants and amplitude in RTN under the various stress regions is investigated and a trap-assisted tunneling model through NBT stress-induced switching traps is proposed to explain the mechanism of $I_{G}$-RTN. The results illustrate that, under the relative high NBT stress, at least some of the holes are discharged from the traps to the gate side.

\section{Experiment}

Plasma nitric oxide (PNO) pMOSFETs used in this study were fabricated with a standard CMOS process technology. The devices size $(L \times W)$ is $30 \mathrm{~nm} \times 70 \mathrm{~nm}$ and dielectric thickness is about $2.6 \mathrm{~nm}$. And the $I_{D}-V_{G}$ curve of this device is shown in the inset of Figure 1. The measurements were conducted using a Keithley 4200 semiconductor characterization 

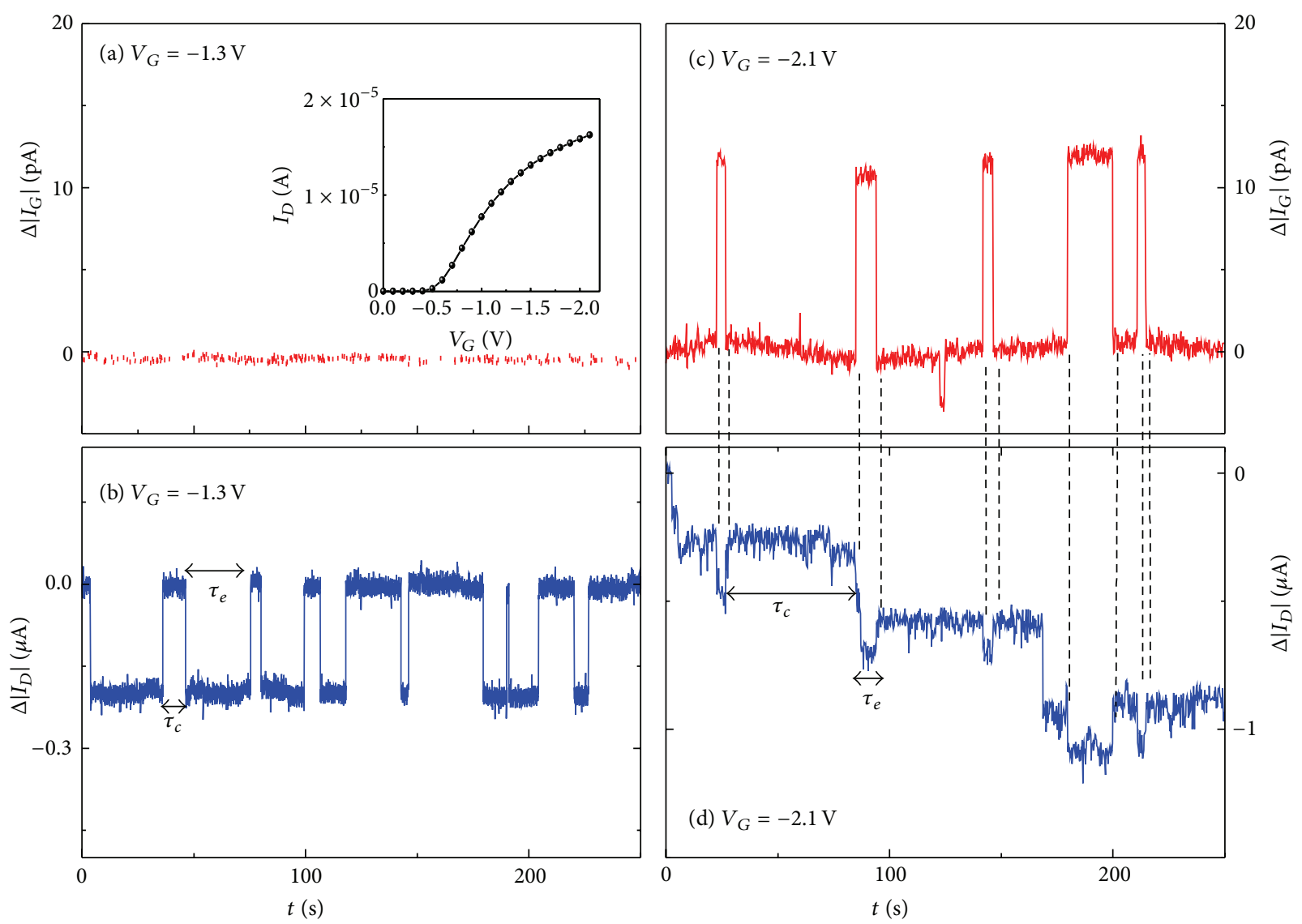

Figure 1: The time trace of $I_{G}$ and $I_{D}$ fluctuation under the gate voltage $V_{G}$ of $-1.3 \mathrm{~V}$ and $-1.9 \mathrm{~V}$ with $V_{\mathrm{DS}}$ of $50 \mathrm{mV}$. The inset shows the $I_{D}-V_{G}$ curve of the experimental device.

system and a Cascade Summit 12000 probe station with a built-in temperature controller.

\section{Result and Discussion}

Figure 1 shows the time traces of $I_{G}$ and $I_{D}$ monitored simultaneously under the relative low $\left(V_{G}=-1.3 \mathrm{~V}, E_{\mathrm{ox}}=\right.$ $3.1 \mathrm{MV} / \mathrm{cm})$ and high $\left(V_{G}=-2.1 \mathrm{~V}, E_{\mathrm{ox}}=6.2 \mathrm{MV} / \mathrm{cm}\right) \mathrm{NBT}$ with $V_{\mathrm{DS}}$ of $50 \mathrm{mV}$ at room temperature stress for pMOSFETs. $I_{D}$-RTN is clearly detected in both cases, which should be contributed by the traps in the gate oxide layer. But there are two major differences between the two stress conditions. One is that the higher stress voltage triggers $I_{G}$-RTN, which has the strong reverse correlation with $I_{D}$-RTN; for example, the low drain current RTN level corresponds to the high gate current RTN level. The correlation suggests that $I_{G}-$ RTN and $I_{D}$-RTN share the same traps induced by NBT stress, or a trapped hole results in the simultaneous increase in $I_{G}$ and decrease in $I_{D}$ under the relatively high NBT stress condition.

By using the carrier separation measurement as shown in Figure 2, the drain and source electrodes were connected together and the gate electrode was biased under the stress condition [15-17]. In that case, the $I_{G}$ current could be divided into hole (source/drain) part and electron (substrate) part. The hole current, electron current, and $I_{G}$ components could

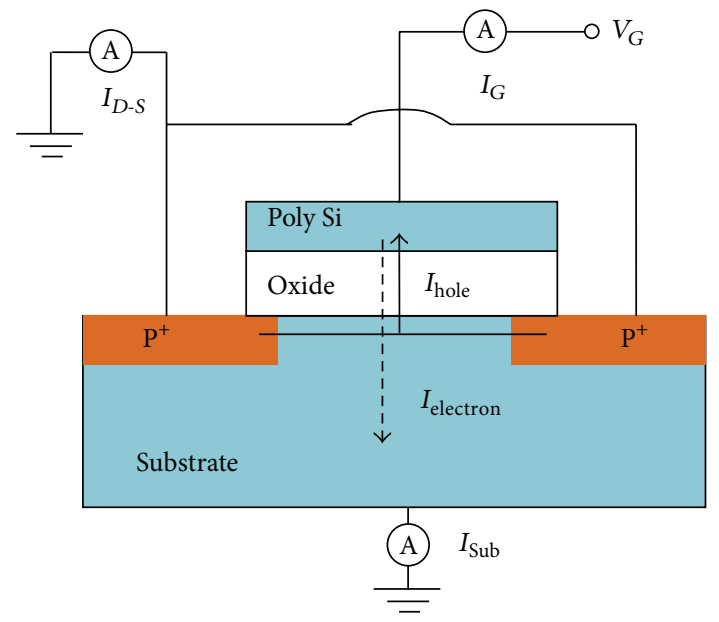

FIGURE 2: Configuration of the circuit diagram in carrier separation measurements. The gate electrode is biased for the stress condition, and drain and source electrodes are connected together.

be monitored simultaneously with the stress time. We could find several RTN fluctuations in the traces of $I_{\text {Hole }}$ and $I_{G}$ and no obvious RTN fluctuations in the $I_{\text {electron }}$ trace as shown in Figure 3. Moreover, it could be seen that the RTN fluctuations in the traces of $I_{\text {Hole }}$ have almost same amplitude 


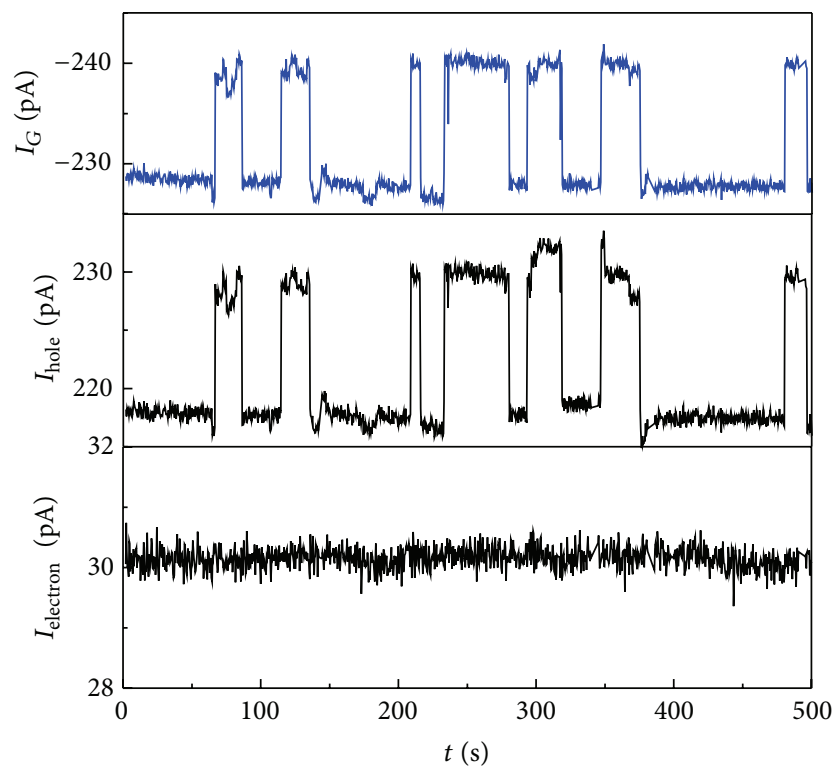

FIGURE 3: The time traces of gate current, electron current, and hole current under NBT stress $(-1.9 \mathrm{~V})$ at $T=320 \mathrm{~K}$ by using carrier separation technique.

and synchronize with that in the $I_{G}$ trace. Therefore, holes are the major contributors to $I_{G}$-RTN. To conclude, the switching hole trap generated in high NBT stress could induce a larger amount of hole from channel to gate.

Another major difference for the two stress conditions is the electrical behaviors of $I_{D}$-RTN. Figure 4 shows the field dependence of the average value of capture time $\left(\tau_{c}\right)$ and emission time $\left(\tau_{e}\right)$ for the $I_{D}$-RTN within the gate voltage of $(-1.0 \mathrm{~V} \sim-1.3 \mathrm{~V})$ and $(-1.8 \mathrm{~V} \sim-2.1 \mathrm{~V})$. The average values of $\tau_{c}$ and $\tau_{e}$ are obtained from the exponential distribution of the single $\tau_{c}$ and $\tau_{e}$. As shown in Figure 4(a), for the low stress range, $\tau_{c}$ and $\tau_{e}$ are found to exponentially decrease and increase with $V_{G}$, respectively. As shown in Figure 4(b), for the high stress range, $\tau_{e}$ keeps weakly dependent with the field even though $\tau_{c}$ exhibits similar behaviours as these observed in the low stress. The strong field dependence of both $\tau_{c}$ and $\tau_{e}$ in $I_{D}$-RTN under low stress could be explained by the extended nonradiative multiphonon (eNMP) theory [18-22]. In this theory, when the negative gate bias is applied for pMOSFETs, the switching trap states can be created from $\mathrm{Si}-\mathrm{Si}$ precursors in the oxide state by capturing a hole via a multiphonon emission (MPE) process $[18,20]$. The increase of the gate field results in a lower MPE barrier. The latter enhances the charge transfer reaction, leading to the dramatic reduction of hole capture time, which could be described as follows [23-25]:

$$
\tau\left(\Delta E_{B}, F_{\mathrm{OX}}, F_{C}\right)=\tau_{0} \cdot \exp \left(\frac{\Delta E_{B}}{K T}\right) \cdot \exp \left(-\frac{F_{\mathrm{ox}}^{2}}{F_{c}^{2}}\right) .
$$

Here $\Delta E_{B}$ is the multiphonon emission (MPE) barrier for hole capture, $F_{\mathrm{ox}}$ is the applied field in the oxide layer, and $F_{c}$ is the characteristic field in MPFAT process. The red solid lines in Figure 4(a) present the fitting results with $F_{c}$ being about $2.5 \mathrm{MV} / \mathrm{cm}$ which is analogous to that measured in NBTI $[20,26]$. On the other hand, increasing the stress field will raise the hole traps' energy level and prevent the hole back to channel, which finally increases the emission time of RTN which is corresponding to the result in the low stress range.

However the electrical behaviour of $\tau_{e}$ in the high stress range is hard to be explained by this theory. One possible explanation is that the hole emission from trap is not to the Si substrate side but to the gate side by tunneling $[27,28]$. In this case, increasing the stress field not only could prevent the hole back to channel but also will encourage it to be emitted to gate, which finally results in a weakly field dependence. Therefore, these switching traps behave as hole current path from channel to gate at high gate bias, which is consistent with the reverse correlation of $I_{D}$ and $I_{G}$-RTN.

For verifying the current path induced by switching traps under high gate bias, the field and temperature dependence of the $I_{G}$-RTN is measured. As shown in the inset of Figure 5, the extremely weak temperature dependence was observed in $\Delta I_{G}$, which is the typical behavior of elastic tunneling. In one-step elastic TAT model, channel charges could tunnel to gate side with the assistance of a trap in oxide. And the TAT probability in the model is described as [27, 29]

$$
\begin{aligned}
I_{\mathrm{TAT}}= & A F_{\mathrm{OX}}^{2} \cdot\left(\frac{\Phi_{T}}{x F_{\mathrm{OX}}}\right) \cdot\left(\frac{2 \Phi_{T}}{x F_{\mathrm{OX}}}-1\right) \\
& \cdot \exp \left(-\frac{8 \pi \sqrt{2 m_{\mathrm{OX}}} \Phi_{T}^{3 / 2}}{3 h q F_{\mathrm{OX}}} \cdot\left[1-\left(1-\frac{x F_{\mathrm{OX}}}{\Phi_{T}}\right)^{3 / 2}\right]\right) .
\end{aligned}
$$

Here $\Phi_{T}$ is the energy depth of the trap from the valence band of dielectric, $m_{\mathrm{ox}}$ is the hole effective mass in dielectric, $F_{\text {ox }}$ is electric field in oxide, and $x$ is the trap's distance from the interface of gate and oxide. When $x=1.5 \mathrm{~nm}$ and $\Phi_{T}=4 \mathrm{~V}$, it is observed that it provides a good description of the experimental data in Figure 5, which verifies that these switching traps could perform as hole tunneling paths.

Finally, the step-like fluctuations detected in Figure 1(b) are investigated. To avoid the mixture of several fluctuations, we selected the device which has only one active trap under the measurement condition. The gate voltage dependence of Id fluctuation of this trap is measured. Under the high bias of $-1.7 \mathrm{~V}$, the emission of the carriers from the trap is not detected until the end of measurement time of $300 \mathrm{~s}$. Therefore the step-like behavior at $-1.7 \mathrm{~V}$ is presented. When the gate bias is reduced from $-1.7 \mathrm{~V}$ to $-1.5 \mathrm{~V}$, the emission of carriers from the trap occurs at about $210 \mathrm{~s}$ and the emission time $\left(\tau_{e}\right)$ is about $170 \mathrm{~s}$. Further decreasing the gate bias reduces the emission time to about $70 \mathrm{~s}$ at $V_{G}=-1.4 \mathrm{~V}$ and about $10 \mathrm{~s}$ at $V_{G}=-1.2 \mathrm{~V}$ (Figure 6). The voltage dependence of the emission time observed here is consistent with the field behaviors of $I_{D}$-RTN in Figure 4(a). The results clearly inform that $I_{D}$-RTN transforms to $I_{D}$-step when the gate bias is increased. In other words, both $I_{D}$-step and $I_{D}$-RTN are originated from the switching traps.

According to the above analyses, $I_{G}$-RTN, $I_{D}$-RTN, and $I_{D}$-step observed in NBTI degradation are due to the generated NBT traps. Under the relatively low NBT stress, the 


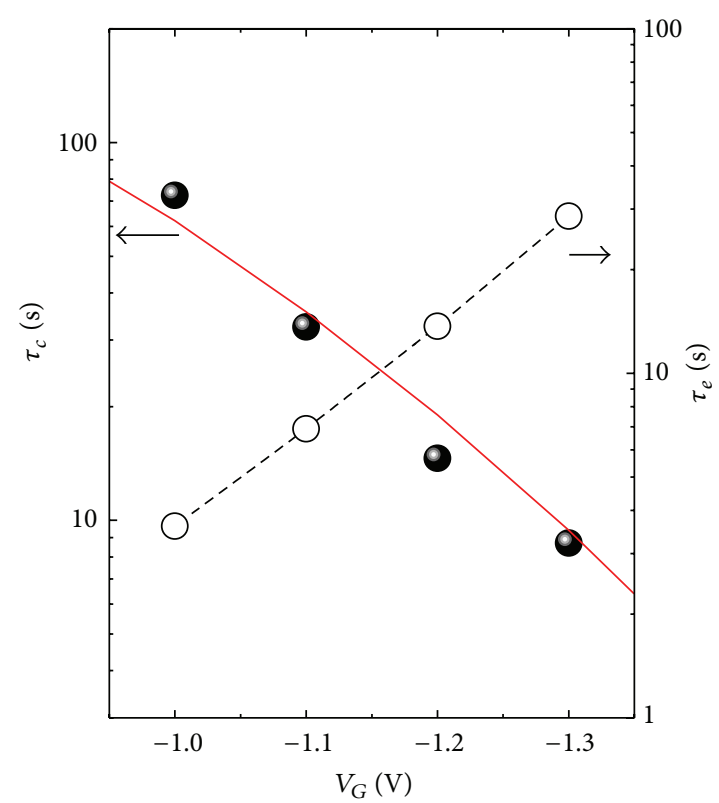

(a)

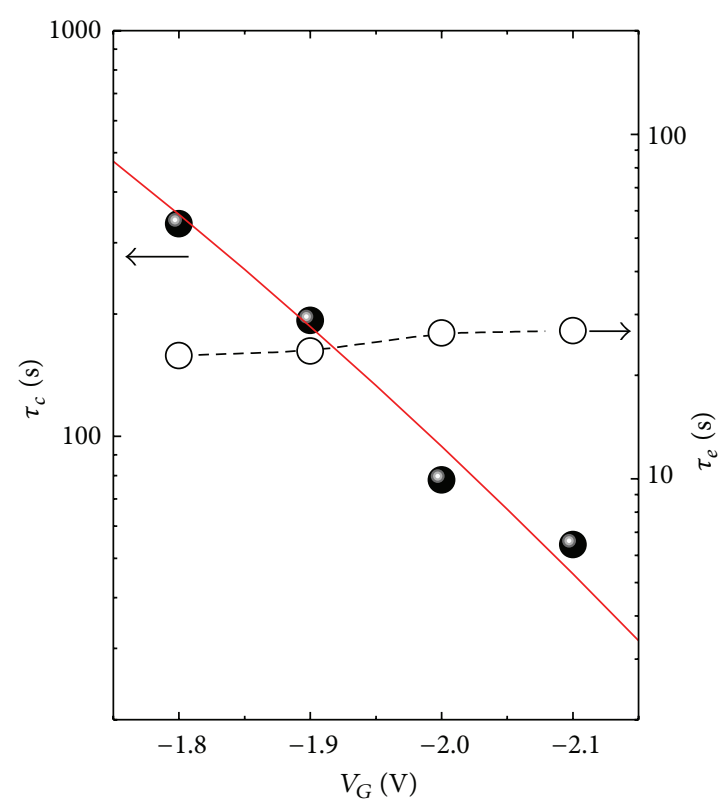

(b)

FIGURE 4: Field dependence of the average value of $\tau_{c}$ and $\tau_{e}$ of the RTN under (a) higher and (b) lower gate bias regions. The red lines present MPFAT simulation results for $\tau_{c}$.

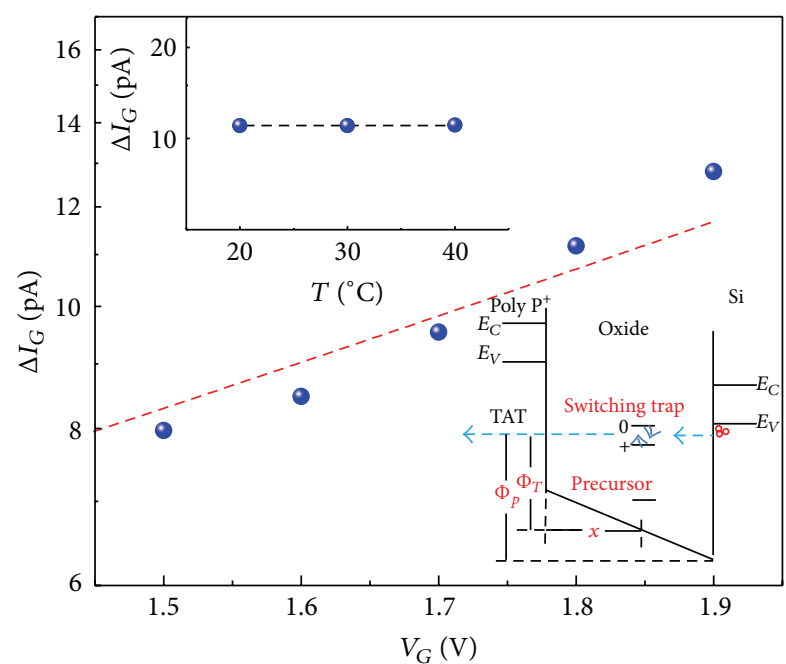

Figure 5: The field dependence of $\Delta I_{G}$ amplitude. The red dash line presents the simulation results by trap-assisted tunneling (TAT) modeling. The inset shows temperature behaviors of $\Delta I_{G}$.

generated traps exchange holes with channel as shown in Figure 7(a), which induces the RTN fluctuations in $I_{D}$. Under the high NBT stress, larger oxide field raises the hole traps' energy level and prevents the hole back to channel. Some traps keep charged state for very long time, as observing steplike behaviors in $I_{D}$, while the other traps emit charged holes to the gate side through TAT process as shown in Figure 7(b). In the latter case, the hole current path from channel to gate is formed. As a result, RTN fluctuations in $I_{G}$ are detected.

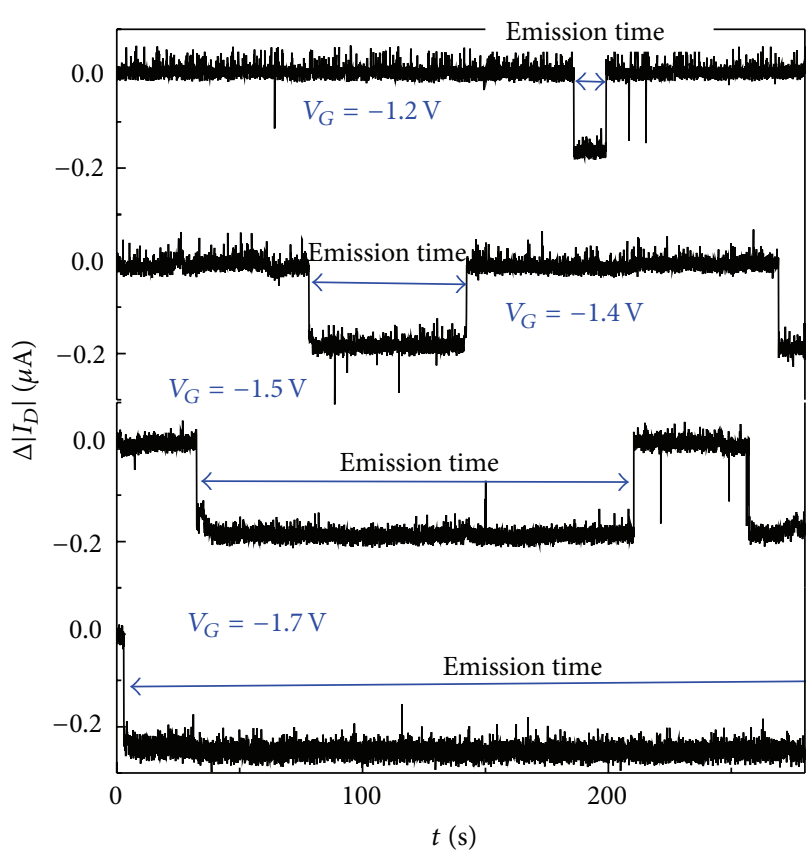

FIGURE 6: The transformation of transience signal from $I_{D}$-Step to $I_{D}$-RTN could be seen by decreasing the gate bias from $-1.7 \mathrm{~V}$ to $-1.5 \mathrm{~V}$. And the emission time of this trap is obviously decreased from larger than $300 \mathrm{~s}$ to about $10 \mathrm{~s}$ when gate bias is decreased from $-1.7 \mathrm{~V}$ to $-1.2 \mathrm{~V}$.

\section{Conclusions}

In this paper, the fluctuations including $I_{G}$-RTN, $I_{D}$-RTN, and $I_{D}$-step are studied under various NBT stress. Note 


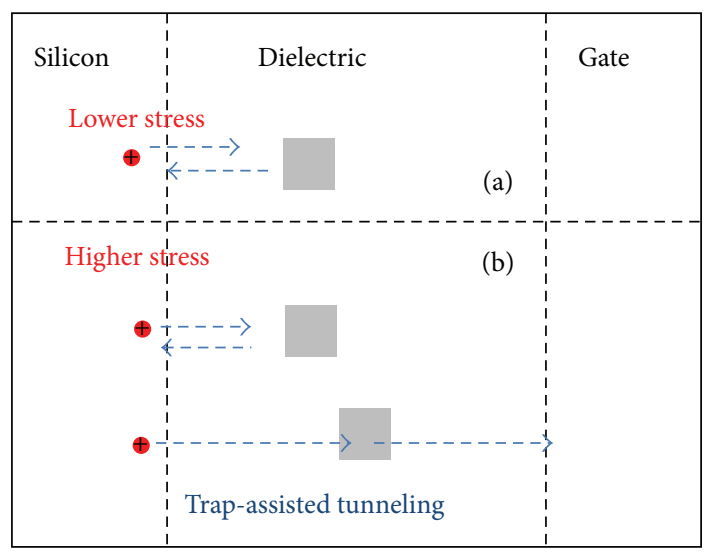

FIGURE 7: Schematic view of the switching traps in pMOSFETs. Under low gate bias, the switching traps in oxide layer could only be recovered by exchanging hole with channel and induce only $I_{D^{-}}$ RTN. Under the high gate bias, part of generated switching traps can capture and hold the hole while some others transform the hole from the channel to gate by TAT mechanism.

that, under the relative low NBT stress, only $I_{D}$-RTN could be detected while under the relative high NBT stress the $I_{D}$-RTN, $I_{G}$-RTN, and $I_{D}$-step are observed. Through the analysis of the field dependence of emission constant and the carrier separation measurement, it is found that under the relative high NBT stress some traps keep charged state for very long time, as observing step-like behaviors in $I_{D}$, while other traps emit charged holes to the gate side through TAT process, which originate both $I_{D}$-step and $I_{D}$-RTN.

\section{Conflict of Interests}

The authors declare that there is no conflict of interests regarding the publication of this paper.

\section{Acknowledgment}

This work was supported by the 973 Program of China (nos. 2010CB934200 and 2012CB619200).

\section{References}

[1] K. O. Jeppson and C. Svennson, "Negative bias stress of MOS devices at high electric fields and degradation of MNOS devices," Journal of Applied Physics, vol. 48, no. 5, pp. 2004-2014, 2004.

[2] S. Ogawa and N. Shiono, "Generalized diffusion-reaction model for the low-field charge-buildup instability at the $\mathrm{Si}_{-} \mathrm{SiO}_{2}$ interface," Physical Review B, vol. 51, p. 4218, 1995.

[3] C. R. Parthasarathy, M. Denais, V. Huard et al., "Designing in reliability in advanced CMOS technologies," Microelectronics Reliability, vol. 46, no. 9-11, pp. 1464-1471, 2006.

[4] H. C. Ma, J. P. Chiu, C. J. Tang, T. Wang, and C. S. Chang, in Proceedings of the International Reliability Physics Symposium, 2009.

[5] D. K. Schroder, "Negative bias temperature instability: what do we understand?" Microelectronics Reliability, vol. 47, no. 6, pp. 841-852, 2007.
[6] V. Huard, C. Parthasarathy, C. Guerin et al., "NBTI degradation: from transistor to SRAM arrays," in Proceedings of the 46th IEEE International Reliability Physics Symposium, pp. 289-300, May 2008.

[7] B. Kaczer, T. Grasser, P. J. Roussel et al., "Origin of NBTI variability in deeply scaled pFETs," in Proceedings of the IEEE International Reliability Physics Symposium, pp. 26-32, May 2010.

[8] M. Toledano-Luque, B. Kaczer, E. Simoen et al., "Correlation of single trapping and detrapping effects in drain and gate currents of nanoscaled nFETs and pFETs," in Proceedings of the IEEE International Reliability Physics Symposium (IRPS '12), pp. XT.5.1-XT.5.6, IEEE, Anaheim, Calif, USA, April 2012.

[9] X. Ji, Y. Liao, C. Zhu et al., "The physical mechanisms of IG random telegraph noise in deeply scaled pMOSFETs," in Proceedings of the IEEE International Reliability Physics Symposium (RPS '13), pp. XT.7.1-XT.7.5, Anaheim, Calif, USA, April 2013.

[10] R. Degraeve, T. Kauerauf, M. Cho et al., "Degradation and breakdown of $0.9 \mathrm{~nm} \mathrm{EOT} \mathrm{SiO}_{2} \mathrm{ALD} \mathrm{HfO}_{2}$ metal gate stacks under positive constant voltage stress," in Proceedings of the IEEE International on Electron Devices Meeting, IEDM Technical Digest, pp. 408-411, Washington, DC, USA, December 2005.

[11] S. Tsujikawa, "SILC and NBTI in pMOSFETs with ultrathin SiON gate dielectrics," IEEE Transactions on Electron Devices, vol. 54, no. 3, pp. 524-530, 2007.

[12] P.-J. Wagner, B. Kaczer, A. Scholten et al., "On the correlation between NBTI, SILC, and flicker noise," in Proceedings of the IEEE International Integrated Reliability Workshop (IIRW '12), pp. 60-64, October 2012.

[13] S. Tsujikawa and J. Yugami, "Evidence for bulk trap generation during NBTI phenomenon in pMOSFETs with ultrathin SiON gate dielectrics," IEEE Transactions on Electron Devices, vol. 53, no. 1, pp. 51-55, 2006.

[14] Y. Gao, D. S. Ang, C. D. Young, and G. Bersuker, "Evidence for the transformation of switching hole traps into permanent bulk traps under negative-bias temperature stressing of high-k P-MOSFETs," in Proceedings of the IEEE International Reliability Physics Symposium, pp. 5A.5.1-5A.5.5, April 2012.

[15] W. Mizubayashi, N. Yasuda, H. Ota et al., "Carrier separation analysis for clarifying leakage mechanism in unstressed and stressed $\mathrm{HfAlO}_{x} / \mathrm{SiO}_{2}$ stack dielectric layers," in Proceedings of the 42nd Annual IEEE International Reliability Physics Symposium, Phoenix, Ariz, USA, April 2004.

[16] Y. Shi, T. P. Ma, S. Prasad, and S. Dhanda, "Polarity dependent gate tunneling currents in dual-gate CMOSFETs," IEEE Transactions on Electron Devices, vol. 45, no. 11, pp. 2355-2360, 1998.

[17] S.-I. Takagi, N. Yasuda, and A. Toriumi, "Expérimental evidence of inelastic tunneling in stress-induced leakage current," IEEE Transactions on Electron Devices, vol. 46, no. 2, pp. 335-341, 1999.

[18] T. Grasser, B. Kaczer, W. Goes, T. Aichinger, P. Hehenberger, and M. Nelhiebel, "A two-stage model for negative bias temperature instability," in Proceedings of the IEEE International Reliability Physics Symposium (IRPS '09), pp. 33-44, IEEE, Montreal, Canada, April 2009.

[19] C. H. Henry and D. V. Lang, "Nonradiative capture and recombination by multiphonon emission in GaAs and GaP," Physical Review B, vol. 15, no. 2, p. 989, 1977.

[20] V. Huard, in Proceedings of the International Reliability Physics Symposium, 2010. 
[21] G. Richard, B. William, B. Kaczer, and T. Grasser, "On the thermal activation of negative bias temperature instability," IIRW Final Report 36, 2009.

[22] T. Grasser, H. Reisinger, P.-J. Wagner, F. Schanovsky, W. Goes, and B. Kaczer, in Proceedings of the IEEE International Reliability Physics Symposium, 2010.

[23] T. Grasser, H. Reisinger, W. Goes et al., "Switching oxide traps as the missing link between negative bias temperature instability and random telegraph noise," in IEEE International Electron Devices Meeting, pp. 1-4, 2009.

[24] S. Makram-Ebeid and M. Lannoo, "Quantum model for phonon-assisted tunnel ionization of deep levels in a semiconductor," Physical Review B, vol. 25, no. 10, pp. 6406-6424, 1982.

[25] S. D. Ganichev, W. Prettl, and I. N. Yassievich, "Deep impuritycenter ionization by far-infrared radiation," Physics of the Solid State, vol. 39, no. 11, pp. 1703-1726, 1997.

[26] X. Ji, Y. Liao, F. Yan, Y. Shi, G. Zhang, and Q. Guo, "The energy distribution of NBTI-induced hole traps in the Si band gap in PNO pMOSFETs," in Proceedings of the IEEE International Reliability Physics Symposium (IRPS '12), XT.12.5, p. XT.12.1, Anaheim, Calif, USA, April 2012.

[27] F. Schuler, R. Degraeve, P. Hendrickx, and D. Wellekens, "Physical charge transport models for anomalous leakage current in floating gate-based nonvolatile memory cells," IEEE Transactions on Device and Materials Reliability, vol. 2, no. 4, pp. 80-88, 2002.

[28] M. O. Andersson, Z. Xiao, S. Norrman, and O. Engström, "Model based on trap-assisted tunneling for two-level current fluctuations in submicrometer metalsilicon-dioxidesilicon diodes," Physical Review B, vol. 41, no. 14, pp. 9836-9842, 1990.

[29] R. Degraeve, F. Schuler, B. Kaczer et al., "Analytical percolation model for predicting anomalous charge loss in flash memories," IEEE Transactions on Electron Devices, vol. 51, no. 9, pp. 13921400, 2004. 

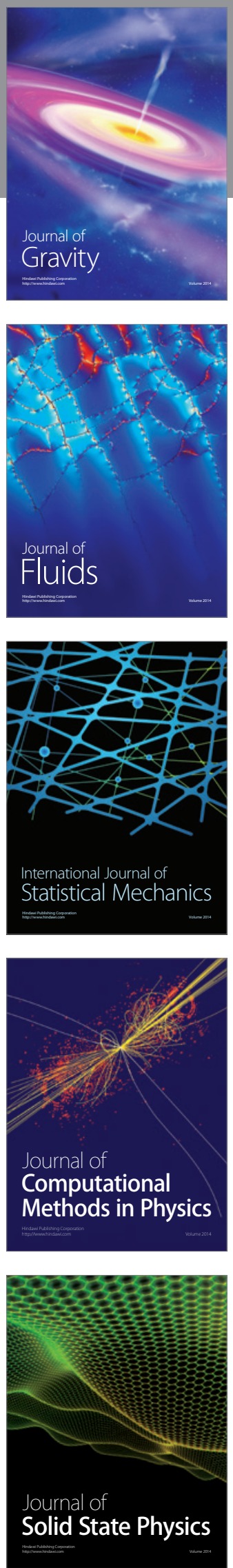

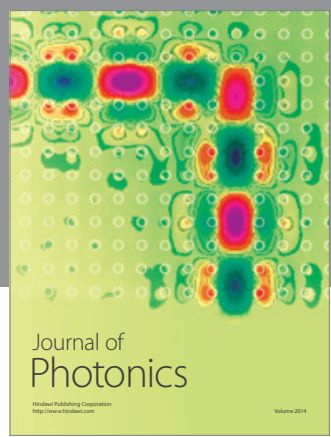

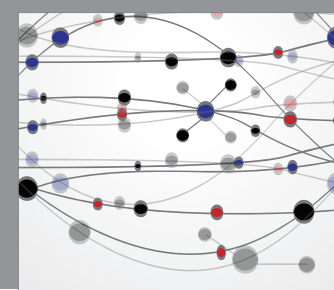

The Scientific World Journal

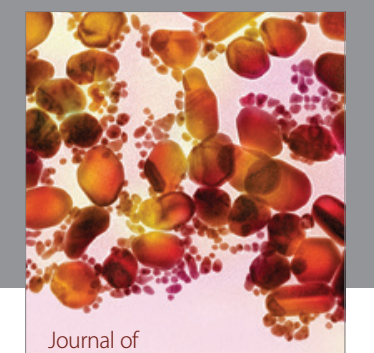

Soft Matter
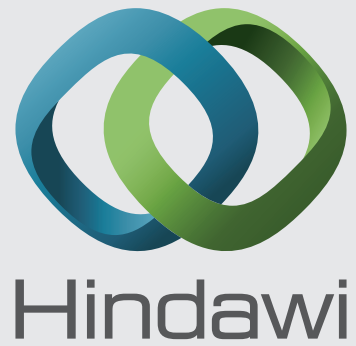

Submit your manuscripts at

http://www.hindawi.com
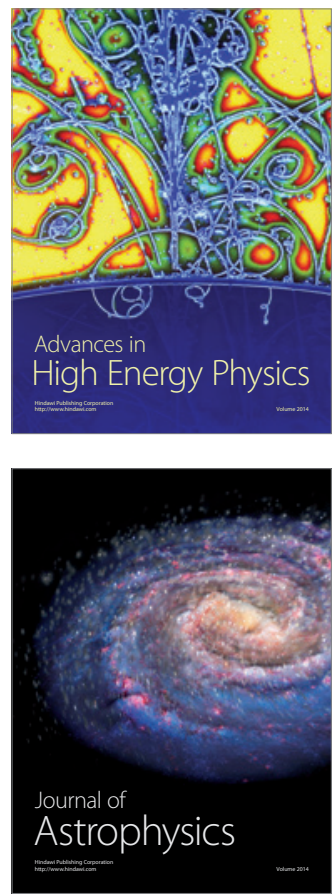
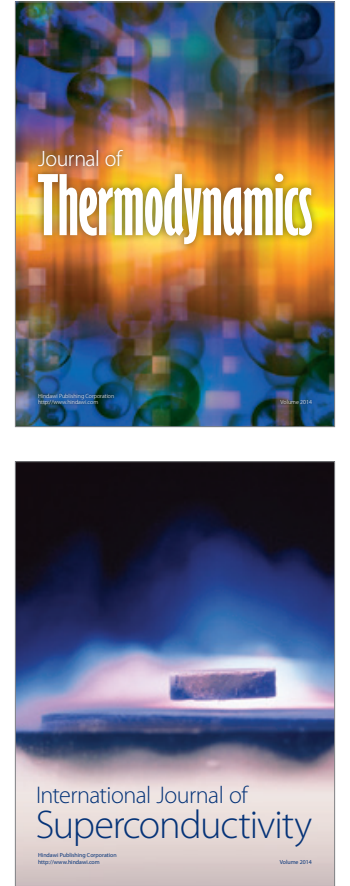
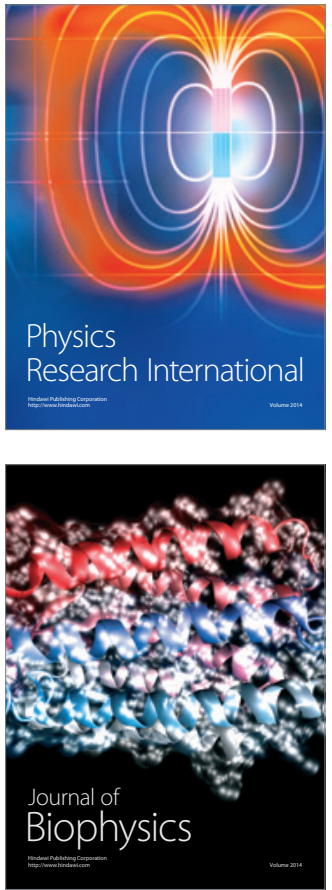
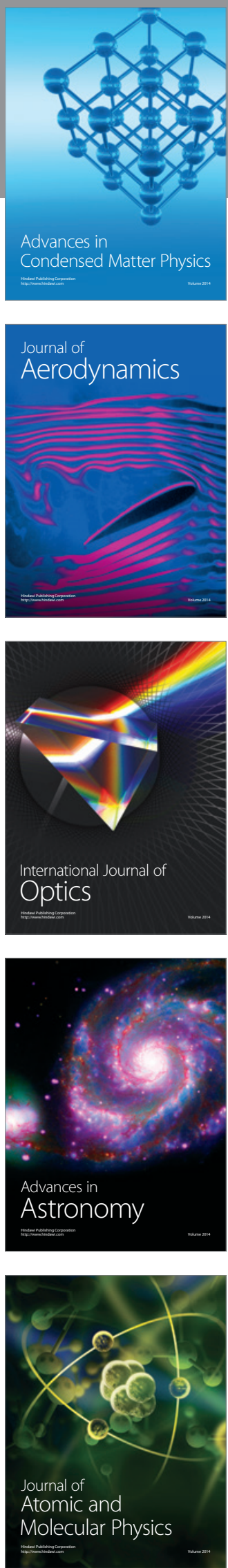